\title{
Microwave Sensing Method for Simultaneous and Independent Determination of Bulk density and Moisture content of Shelled Peanuts
}

\author{
Samir Trabelsi* and Stuart O. Nelson
}
U. S. Department of Agriculture, Agricultural Research Service, P.O. Box 5677, Athens, GA 30604-5677; phone: +1 706546 3157; fax: +1 706546 3607; e-mail: strabelsi@qaru.ars.usda.gov

\section{Introduction}

Rapid nondestructive sensing of physical properties such as bulk density and moisture content in shelled peanuts is important for decision-making during their handling, processing and storage. Dielectric-based methods have been used successfully for the characterization of cereal grain and oilseed [1-3]. In this paper, a method is used to predict bulk density and moisture content in shelled peanuts from measurement of their dielectric properties. The method consists of establishing direct relationships between the dielectric properties and bulk density and moisture content. This method permits simultaneous determination of bulk density and moisture content. It is based on a complex-plane representation [4] from which the bulk density can be determined from the dielectric properties independent of temperature and moisture content. Then moisture content is determined independent of bulk density by means a permittivity calibration function that is expressed in terms of measured dielectric properties [5]. Bulk density and moisture calibration equations are given at $24{ }^{\circ} \mathrm{C}$ and $\mathrm{X}$-band microwave frequencies ranging from $8 \mathrm{GHz}$ to $12 \mathrm{GHz}$.

\section{Approach}

The dielectric properties of shelled peanuts were measured in free space with a pair of horn/lens antennas and an HP $8510 \mathrm{C}$ vector network analyzer at $24{ }^{\circ} \mathrm{C}$ between 8 and 12 GHz. The measurement method is described in detail elsewhere [6]. The sample consisted of $7 \mathrm{~kg}$ of Runner type peanuts, cv. Georgia Green. Starting with a relatively low moisture content, the moisture content was increased gradually in increments of about 1\% until the higher end of the desired moisture range was reached. For each moisture level, the dielectric properties measurements were performed at three different densities ranging from loosely packed to well settled. In this study, the moisture content, $M$, ranged from $6.6 \%$ to $17.2 \%$, the bulk density, $\rho$, ranged from $0.60 \mathrm{~g} / \mathrm{cm}^{3}$ to 0.73 $\mathrm{g} / \mathrm{cm}^{3}$, and temperature, $T$, was about $24{ }^{\circ} \mathrm{C}$.

The dielectric properties of shelled peanuts were computed from the measurement of the modulus, $\left|S_{21}\right|$, and phase, $\varphi$, of the complex transmission scattering coefficient, as follows:

$$
\begin{aligned}
& \varepsilon^{\prime}=\left[1-\frac{(\varphi-360 n)}{360 d} \frac{c}{f}\right]^{2} \\
& \varepsilon^{\prime \prime}=\frac{-20 \log \left|S_{21}\right|}{8.686 \pi d} \frac{c}{f} \sqrt{\varepsilon^{\prime}}
\end{aligned}
$$


where $c$ is the speed of light in $\mathrm{m} / \mathrm{s}, f$ is the frequency in $\mathrm{Hz}, d$ is the thickness of the layer of material in meters, and $n$ is an integer to be determined [7]. The two components of the complex permittivity, as given in equations (1) and (2) are the average values for the whole sample.

\section{Results}

Use of complex-plane representation for bulk density determination. The complexplane representation (Argand diagram) of the dielectric properties, also known as a ColeCole plot, is often used to analyze the behavior of dielectric materials [8]. Instead of plotting $\varepsilon^{\prime \prime}$ versus $\varepsilon^{\prime}$ here, the dielectric loss factor divided by bulk density is plotted against the dielectric constant divided by bulk density for samples of different moisture contents. Fig. 1 shows the complex-plane presentation for shelled peanuts at frequencies between 8 and $12 \mathrm{GHz}$. In this representation, data collected at different moisture contents and bulk densities are aligned along the same straight line [9] with data corresponding to higher moisture contents at higher coordinates. The $\mathrm{x}$-axis intercept is common for all frequencies. The data shown in Fig. 1 can be fitted by a linear regression of the form:

$$
\frac{\varepsilon^{\prime \prime}}{\rho}=a_{f}\left(\frac{\varepsilon^{\prime}}{\rho}-k\right)
$$

where $a_{f}$ is the slope of the line and $k$ is the $\mathrm{x}$-axis intercept. Table 1 provides the regression coefficients and coefficients of determination, $r^{2}$, at several frequencies. Table 2 indicates that the slope, $a_{f}$, increases with frequency and that the $\mathrm{x}$-axis intercept is nearly the same at all frequencies. There exists a linear relationship between $a_{f}$ and frequency:

$$
a_{f}=0.045 f+0.351 \quad r^{2}=0.993
$$

where $f$ is the frequency in GHz. At a given frequency, bulk density can be determined directly from measurement of the dielectric properties by solving equation (3) for $\rho$ :

$$
\rho=\frac{a_{f} \mathcal{E}^{\prime}-\varepsilon^{\prime \prime}}{k a_{f}}
$$

Equation (5) indicates that the bulk density of a given sample of shelled peanuts can be determined without knowledge of moisture content and temperature of the sample. Values of the standard error of calibration $(S E C)$ for bulk density determination with (5) are given in Table 1 at several frequencies. They are the same at all frequencies.

Use of density-independent permittivity function for moisture determination. In many instances, moisture content in granular materials has to be determined without knowledge of bulk density. A density-independent permittivity function was used successfully for moisture determination in cereal grain and oilseed. It is expressed in terms of $\varepsilon^{\prime}, \varepsilon^{\prime \prime}$ and $a_{f}$ as follows [5]:

$$
\psi=\frac{\varepsilon^{\prime \prime}}{\varepsilon^{\prime}\left(a_{f} \varepsilon^{\prime}-\varepsilon^{\prime \prime}\right)}
$$




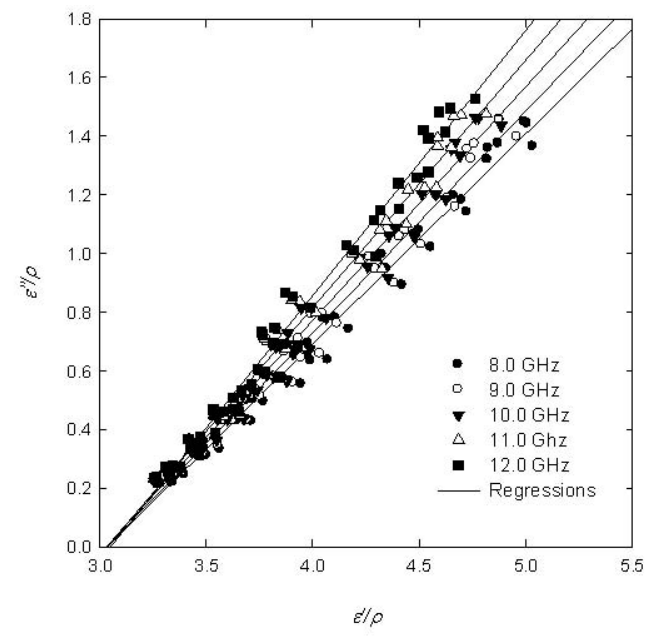

Fig. 1 Complex-plane representation of the dielectric constant divided by density and loss factor divided by density at $24{ }^{\circ} \mathrm{C}$ and indicated frequencies.

Table 1. Regression statistics corresponding to equations (3) to (5) and the SEC for bulk density determination of shelled peanuts at different frequencies and $24{ }^{\circ} \mathrm{C}$.

\begin{tabular}{|c|c|c|c|c|c|}
\hline Statistics & $8 \mathrm{GHz}$ & $9 \mathrm{GHz}$ & $10 \mathrm{GHz}$ & $11 \mathrm{GHz}$ & $12 \mathrm{GHz}$ \\
\hline$a_{f}$ & 0.720 & 0.759 & 0.795 & 0.842 & 0.902 \\
\hline$k$ & 3.04 & 3.04 & 3.03 & 3.03 & 3.05 \\
\hline$r^{2}$ & 0.983 & 0.982 & 0.982 & 0.980 & 0.979 \\
\hline$S E C_{\rho}\left(\mathrm{g} / \mathrm{cm}^{3}\right)$ & 0.014 & 0.014 & 0.014 & 0.014 & 0.014 \\
\hline
\end{tabular}

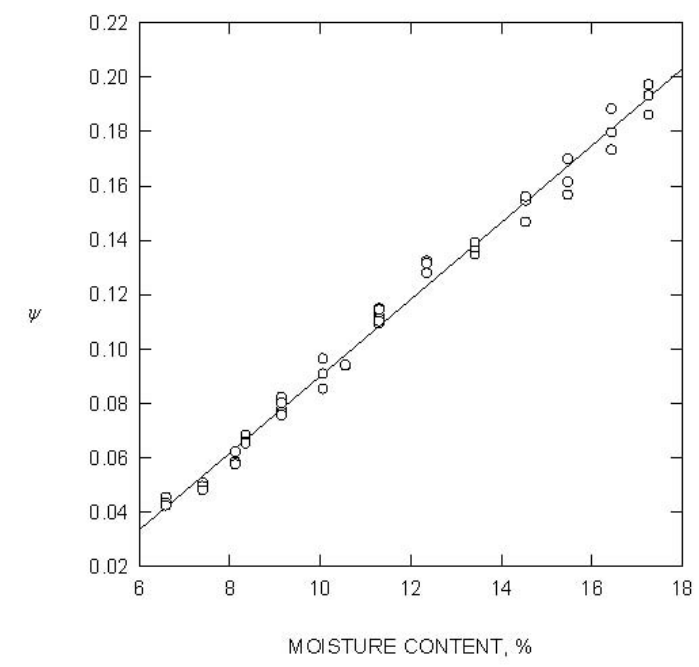

Fig.2 Density-independent permittivity function $\psi$ vs. moisture content of peanut kernels at $10.0 \mathrm{GHz}$ and $24{ }^{\circ} \mathrm{C}$. 
Table 2. Regression statistics corresponding to equations (7) and (8) and the SEC for moisture content determination in shelled peanuts at different frequencies and $24{ }^{\circ} \mathrm{C}$.

\begin{tabular}{llllll}
\hline Statistics & $8 \mathrm{GHz}$ & $9 \mathrm{GHz}$ & $10 \mathrm{GHz}$ & $11 \mathrm{GHz}$ & $12 \mathrm{GHz}$ \\
\hline$a$ & 0.015 & 0.014 & 0.014 & 0.013 & 0.013 \\
$b$ & -0.058 & -0.053 & -0.051 & -0.051 & -0.051 \\
& & & & & \\
$r_{\psi}^{2}$ & 0.977 & 0.991 & 0.990 & 0.988 & 0.984 \\
$S E C_{\psi}(\%)$ & 0.28 & 0.30 & 0.32 & 0.36 & 0.41 \\
\hline
\end{tabular}

Fig. 2 shows the dependence of $\psi$ on moisture content at $10 \mathrm{GHz}$ and $24{ }^{\circ} \mathrm{C}$. Densityindependent $\psi$ increases linearly with moisture content. Therefore, a linear regression can be used to fit the data:

$$
\psi=a M+b
$$

Regression constants for this equation and corresponding coefficients of determination are given in Table 2. The moisture calibration equation can be determined from equation (7) as follows:

$$
M=\frac{\psi-b}{a}
$$

The SEC values corresponding to moisture calibration equation (8) are given in Table 2.

\section{References}

[1] A. Kraszewski, Microwave Aquametry. Piscataway, NJ: IEEE Press, 1996.

[2] S. Trabelsi, A. W. Kraszewski, and S. O. Nelson, "Determining physical properties of grain by microwave permittivity measurements," Transactions of the ASAE, vol. 42, pp. 531-536, 1999.

[3] S. Trabelsi and S. Nelson, "Calibration methods for nondestructive microwave sensing of moisture content and bulk density of granular materials," Transactions of the ASAE, vol. 47, pp. 1999-2008, 2004.

[4] S. Trabelsi, A. Kraszewski, and S. Nelson, "Microwave dielectric sensing of bulk density of granular materials," Measurement Science and Technology, vol. 12, pp. 2192-2197, 2001.

[5] S. Trabelsi, A. Kraszewski, and S. O. Nelson, "New density-independent calibration function for microwave sensing of moisture content in particulate materials," IEEE Transactions on Instrumentation and Measurement, vol. 47, pp. 613-622, 1998.

[6] S. Trabelsi and S. O. Nelson, "Free-space measurement of dielectric properties of cereal grain and oilseed at microwave frequencies," Measurement Science and Technology, vol. 14, pp. 589-600, 2003.

[7] S. Trabelsi, A. W. Kraszewski, and S. O. Nelson, "Phase-shift ambiguity in microwave dielectric properties measurements," IEEE Transactions on Instrumenation and Measurement, vol. 49, pp. 56-60, 2000.

[8] J. B. Hasted, Aqueous Dielectrics. London: Chapman and Hall, 1973.

[9] S. Trabelsi, A. Kraszewski, and S. O. Nelson, "New calibration technique for microwave moisture sensors," IEEE Transactions on Instrumentation and Measurement, vol. 50, pp. 877-881, 2001. 